\title{
Electrophysiological Recordings on a Sounding Rocket: Report of a First Attempt Using Xenopus laevis Oocytes
}

\author{
Simon L. Wuest ${ }^{1,2}$, Tobias Plüss ${ }^{1}$, Christoph Hardegger ${ }^{1}$, Mario Felder ${ }^{1}$, Aaron Kunz ${ }^{1}$, Benno \\ Fleischli ${ }^{1}$, Carlos Komotar ${ }^{1}$, Lukas Rüdlinger ${ }^{1}$, Andreas Albisser ${ }^{1}$, Thomas Gisler ${ }^{1}$, Daniela A. \\ Frauchiger $^{2}$, and Marcel Egli ${ }^{1}$ \\ ${ }^{1}$ Lucerne School of Engineering and Architecture, Lucerne University of Applied Sciences and Arts, Horw, \\ Switzerland; ${ }^{2}$ Institute for Surgical Technology \& Biomechanics, University of Bern, Bern, Switzerland
}

\begin{abstract}
It is not fully understood how cells detect external mechanical forces, but mechanosensitive ion channels play important roles in detecting and translating physical forces into biological responses (mechanotransduction). With the "OoClamp" device, we developed a tool to study electrophysiological processes, including the gating properties of ion channels under various gravity conditions. The "OoClamp" device uses an adapted patch clamp technique and is operational during parabolic flight and centrifugation up to $20 \mathrm{~g}$. In the framework of the REXUS/BEXUS program, we have further developed the "OoClamp" device with the goal of conducting electrophysiological experiments aboard a flying sounding rocket.
\end{abstract}

Key words: $\quad$ Electrophysiology; Microgravity; Xenopus laevis Oocytes; Sounding Rocket; Mechanobiology; Mechanosensitive Ion Channels

Correspondence to: $\quad$ Marcel Egli

Lucerne School of Engineering and

Architecture, Institute of Medical

Engineering

Seestrasse 41

CH-6052 Hergiswil

Switzerland

Telephone: +41413493618

E-mail: marcel.egli@hslu.ch
The aim of such an experiment was first to assess whether electrophysiological measurements of Xenopus laevis oocytes can be performed on sounding rocket flights, something that has never been done before. Second, we aimed to examine the gating properties of ion channels under microgravity conditions. The experiment was conducted in March 2016 on the REXUS 20 rocket. The postflight analysis showed that all recording chambers were empty as the rocket reached the microgravity phase. A closer analysis of the flight data revealed that the oocytes were ripped apart a few seconds after the rocket launch. This first attempt at using sounding rockets as a research platform for electrophysiological recordings was therefore limited. Our modified "OoClamp" hardware was able to perform the necessary tasks for difficult electrophysiological recordings aboard a sounding rocket; however, the physical stresses during launch (acceleration and vibrations) did not support viability of Xenopus oocytes.

\section{INTRODUCTION}

Biological cells continuously sense and respond to their mechanical environment. To date, several mechanisms have been proposed for how cells detect mechanical forces and induce a corresponding intracellular signal (a 
process called mechanotransduction) (Eyckmans et al., 2011). As an omnipresent force, gravity has a major impact on the evolution and daily life of living organisms. Although gravity is a very small force at the cellular level, gravity variation has profound effects on cells (Pietsch et al., 2011). For example, gravity affects the fluidity of lipid membranes (Sieber et al., 2014) and impacts multiple cellular functions that depend on ion channels (Goldermann and Hanke, 2001; Hanke et al., 2006; Hanke et al., 2002; Kohn, 2013; Meissner and Hanke, 2005; Wiedemann et al., 2002; Wiedemann and Hanke, 2002).

Oocytes from the organism Xenopus laevis are widely used for electrophysiological experiments. Since they are relatively easy to maintain, do not require a sterile environment, and are quite large (approximately $1 \mathrm{~mm}$ in diameter), these oocytes are easy to manipulate. In addition, they can be engineered to heterologously overexpress different membrane proteins, such as ion channels, by microinjection of either RNA or DNA. Due to their robustness, these oocytes have been used successfully in parabolic flights (Richard et al., 2012; Schaffhauser et al., 2011; Wuest et al., 2017). Native oocytes from Xenopus laevis, as well as oocytes that overexpress epithelial sodium channels, demonstrated reduced membrane conductivity under microgravity and increased conductivity under hypergravity (parabolic flights) (Richard et al., 2012; Schaffhauser et al., 2011). This effect is likely due to the gravity-dependent open state probability of epithelial sodium channels and ion channels that are expressed in native oocytes.

In this study, we assessed whether Xenopus laevis oocytes are suitable for electrophysiological measurements during sounding rocket flights. Because sounding rockets experience longer durations of microgravity than parabolic flights, gating properties of specific mechanosensitive ion channels can be studied for minutes, rather than for several seconds, which is the limit of parabolic flights. For this experiment, we further developed our previously introduced "OoClamp" for use during parabolic flights (Schaffhauser et al., 2011).

\section{MATERIALS AND METHODS}

\section{Recording Principle and System Overview}

The hardware was based on a previously published principle (Schaffhauser et al., 2011; Wuest et al., 2017). However, it had to be built from scratch in order to meet the specific requirements for sounding rocket flights and the high integration demands. The recording principle can be thought of as an adapted patch clamp technique (Schaffhauser et al., 2011). The voltage-dependent transmembrane currents of overexpressing or native oocytes were measured in specific recording chambers, each holding one oocyte. Figure 1 illustrates that by pressing the oocyte against an aperture, a patch of the cell membrane could be isolated electrically. The recording chamber was divided into two compartments, separated by the membrane patch. Both compartments were filled with culture medium (see below) and were equipped with two electrodes that allowed for the simultaneous application of a defined voltage $\left(V_{c}\right)$ across the cell patch, and measurement of the corresponding voltage-dependent current.

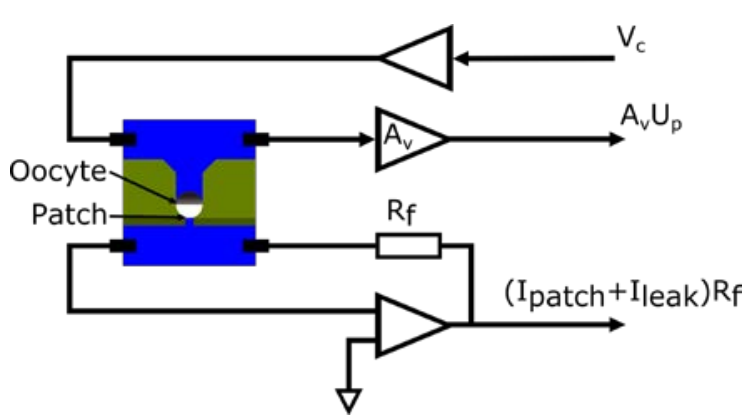

Figure 1. Schematic of the recording principle. An oocyte was placed in the cavity of a silicone chip (light green) and pressed against a small aperture at the lower end, which electrically isolated a patch of the cell membrane. The silicone chip was stabilized with a glass slide (dark green). With the help of four electrodes, a defined voltage was applied across the membrane patch and the corresponding current was measured. The medium in the lower compartment was exchangeable to allow application of different media or drugs.

Compared to conventional patch clamp technique, the oocyte was not impaled with microelectrodes, which significantly simplified 
the experimental design and handling. As a consequence, direct control and measurement of the cytosolic potential was not possible. The contact quality of the oocyte with the aperture was monitored by determining the seal resistance, which was measured by applying one or more (trans-oocyte) voltage steps and simultaneously recording the corresponding currents. The slope of the voltage-current relationship represented the seal resistance and was typically around $100 \mathrm{k} \Omega$.

The medium in contact with the membrane patch could be exchanged, which allowed us to apply and remove specific ion channel blockers. Medium exchange was driven by air pressure. Using a pressure controller to pressurize medium containers, medium was pressed through capillaries into the recording chambers. Valves before the recording chamber allowed for the medium flow to be controlled, and the used medium was collected in a waste bag. To prevent oocytes from being displaced and to ensure good electrical isolation of the membrane patch from the remaining cell membrane (seal), gentle pressure was applied to the upper compartment. Additionally, through a valve, the top pressure could be turned on and off. This allowed for the isolation of a recording chamber from the remaining system in case of malfunction. The voltage and current signals were generated, measured, and digitized in close proximity to the recording chambers.

In total, six recording chambers, each accommodating one oocyte, were installed and operated in parallel. The recording chambers were mounted in two so-called late access modules, each of which had three recording chambers. These late access modules also hosted the electronics necessary for taking measurements, as well as the tubing, capillaries, and valves. From the measurement electronics, the data were sent to the board computer, where they underwent preliminary processing before being stored on a nonvolatile memory device. Figures 2 and 7 illustrate schematic overviews of the system.

\section{Experimental Design}

The purpose of this experiment was to assess if Xenopus laevis oocytes are suitable for electrophysiological measurements during sounding rocket flights. To test this, we required that: (1) the medium in contact with the membrane patch could be exchanged in flight, and (2) the contact between the oocyte and the silicone chip remained stable. We tested medium exchange by switching from a regular physiological solution to a cation-low solution. This provoked a small and unspecific reaction from the oocytes, which could be detected in the current signal (see section "Chemicals and Oocytes" below for more details). The quality of the contact between the oocyte and the silicone chip was tested by measuring the seal resistance (slope of the voltage-current relationship). A seal resistance in the order of $100 \mathrm{k} \Omega$ indicated a good contact.

\section{Mechanics}

We developed a structure that organized the experiment across four decks, as illustrated in Figure 3. Two gas cartridges, as well as pressure controllers and containers for fresh and waste medium, were mounted on the lowest deck. The gas cartridges contained pressurized air at 2.4 bar(a), which was required to drive medium through the recording chambers. The two middle decks were designed to hold the two previously mentioned late access modules. The board computer and associated electronics were installed on the top deck.

\section{Recording chambers}

The core measurements of the experiment were conducted in the recording chambers, designed similar to those previously published (Wuest et al., 2017). Briefly, as illustrated in Figures 1 and 4, each recording chamber held one oocyte and allowed the exchange of medium in contact with the patch of the cell membrane. The oocyte was placed in a cavity of a silicone chip with a tiny hole at the far end, which was reinforced with a glass slide (Figure 4). By applying top pressure, the oocyte was pressed against this hole and a patch of the cell membrane was isolated electrically. Medium exchange was accomplished using a micro-fluidic chip, which was also cast in silicone. Unlike previous experiments (Schaffhauser et al., 2011; Wuest et al., 2017), recording chambers were equipped with a temperature sensor. 


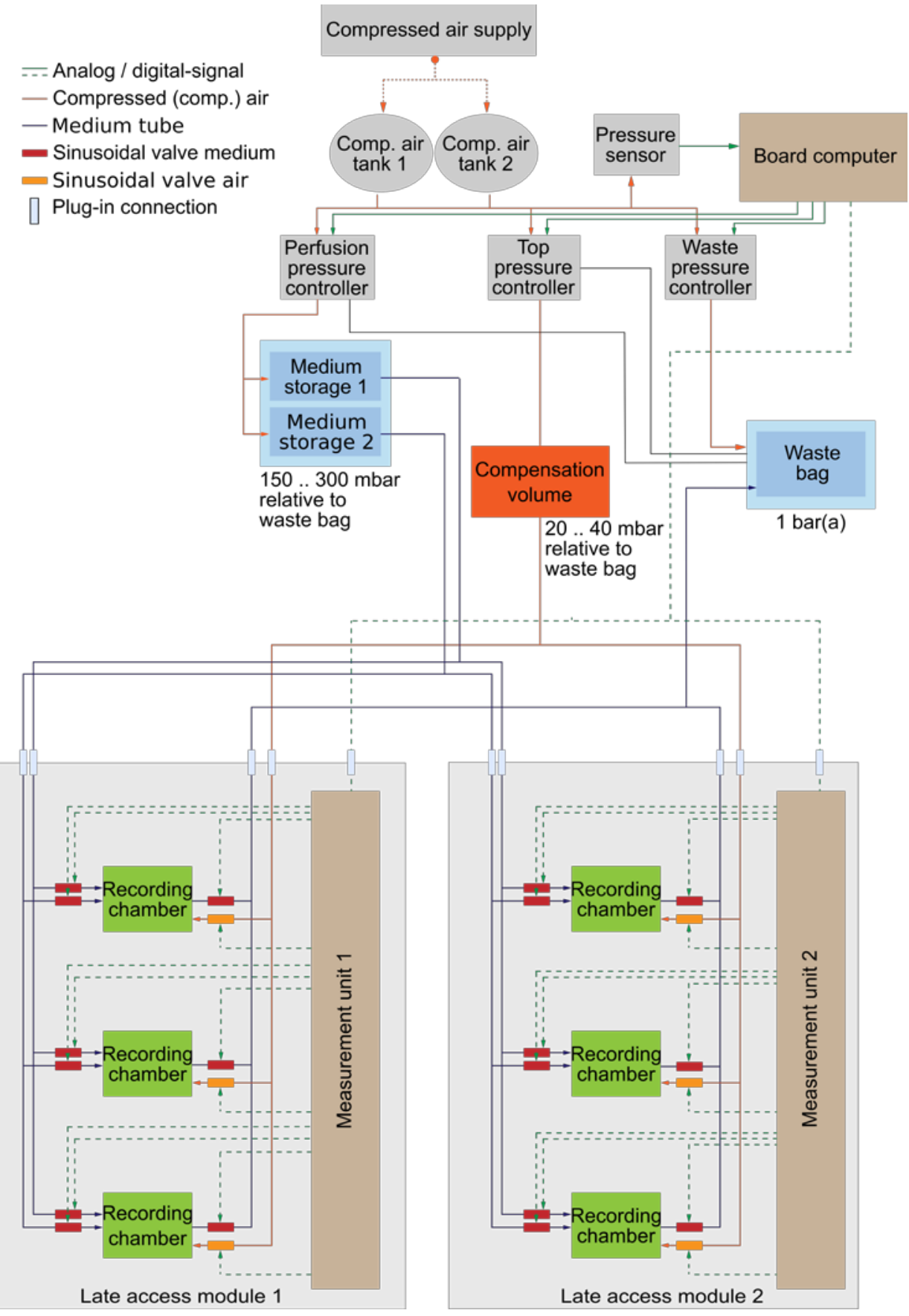

Figure 2. Schematic of the air and liquid system. Air pressure from the reservoir was reduced and regulated to the appropriate level by three pressure controllers: perfusion, top, and waste pressure. The perfusion pressure was used for the flow of the medium through the recording chambers, the top pressure ensured proper placement of the oocytes, and the third pressure controller released the pressure from the waste container as medium was transferred. Fresh medium was distributed from medium container to the two late access modules. In the late access module, the medium lines were split further, leading to valves that controlled the flow of the medium through the chambers. The medium passed through capillaries before reaching the recording chambers, which ensured a constant flow (not shown in the illustration). After the recording chamber, the medium passed through a waste medium valve, which was closed during launch and in case of malfunction. Then, the waste medium was brought to an intermediate waste medium container before leaving the late access module (not shown in schematic). From there, the medium was finally collected in a waste medium bag. 


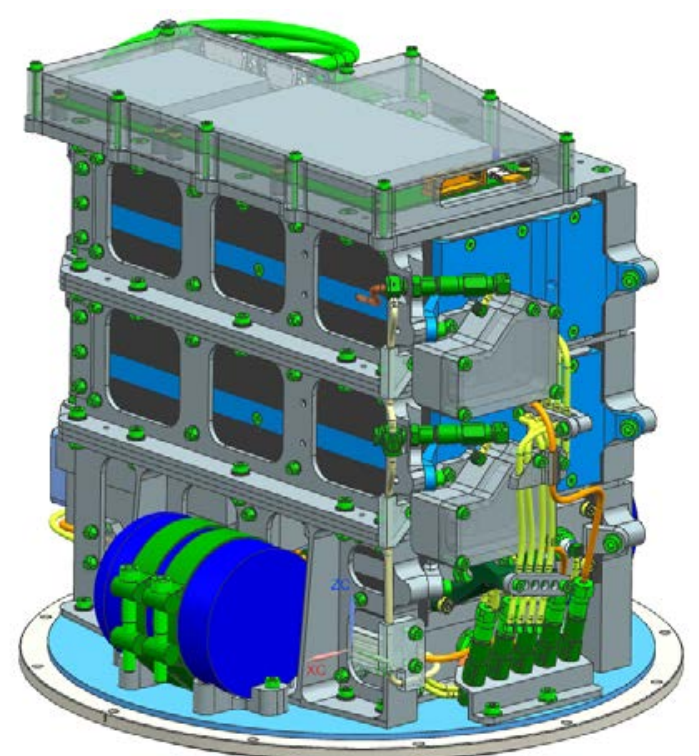

Figure 3. Schematic illustrating the structure of the experiment. The structure organized the experiment across four decks. Two gas cartridges, pressure controllers, and containers for fresh and waste medium were mounted on the lowest deck. Two late access modules were placed on the two middle decks, and three recording chambers were placed in each. The board computer and associated electronics were mounted on the top deck.

During the hardware development process, a problem arose because the hole in the glass slide had sharp edges that destroyed the oocytes during the vibration tests. To avoid the sharp edges, a ruby bearing (developed in the watchmaking industry and obtained from Maillard-Frères SA, Courtemaîche, Switzerland) was used to form the hole. The ruby bearing had an inner diameter of $0.25 \mathrm{~mm}$ and an outer diameter of $2 \mathrm{~mm}$. One side of the bearing (perpendicular to hole) was flat, while the other had a concave indentation. The bearing was glued into a $2 \mathrm{~mm}$ hole in a predrilled glass slide, such that the flat side of the bearing was aligned to the bottom side of the glass slide. Subsequently, silicone was cast around the glass slides (Wuest et al., 2017).

\section{Late access module}

The late access module accommodated three recording chambers, as well as valves, capillaries, and the measuring electronics, illustrated in Figures 2 and 6. Two late access modules were operating in parallel during the experiment. The recording chambers were not fixed; instead, they were embedded in the module in soft foam to decouple the vibration transfer from the module to the recording chambers. The foam was designed in multiple layers, and the tubes and cables were laid between these layers.

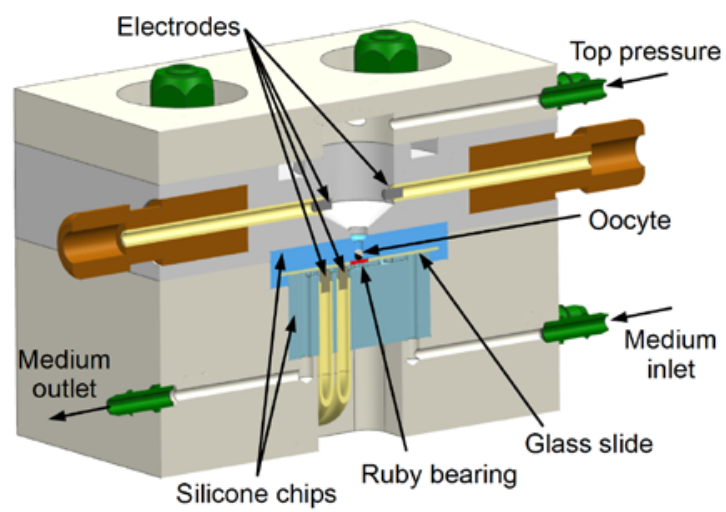

Figure 4. Cross-section view of the recording chamber. The recording chamber accommodated one oocyte, allowing the medium in contact with the patch of the cell membrane to be exchanged, and providing the mechanical support for the electrodes and connecting tubes. The oocyte (black and white) was pressed in a cavity of a silicone chip (light blue), which had a tiny hole at the lower end. The chip was reinforced with a glass slide (gray), and the tiny hole was formed with a ruby bearing (red). The exchange of the medium in contact with the membrane patch was accomplished with a micro-fluidic chip (dark blue). A polymethyl methacrylate (PMMA) housing held the two silicone chips, the electrodes, and the tubing for the fluids in place.

The measurement electronics printed circuit board (PCB) was mounted next to the recording chambers. The valves, tubes, and capillaries to regulate the medium in the recording chambers were installed on the bottom. Four valves were installed for each chamber. Among these four, two normally closed valves allowed for the controlled flow of two different media. The top pressure was switched off using a valve normally left open. A third valve (normally left closed) controlled the media flow through the waste tube. The valves for the top pressure and 
the waste media were closed only during launch and in case of malfunction (e.g., death of the oocyte). This allowed for a recording chamber to be isolated from the other chambers. The late access modules were closed with bottom and top covers and were made out of aluminum, thus also providing electrical shielding for the sensitive measurements.

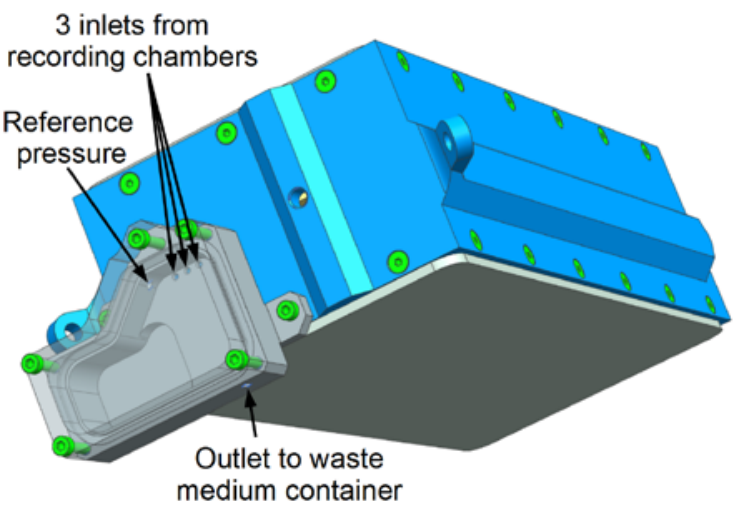

Figure 5. Schematic of the intermediate waste medium container. To ensure a constant pressure at the recording chamber outlet, an intermediate waste medium container was mounted on the front side of the late access module. The waste lines from the recording chambers entered the upper part of this container. A further connection enabled fast pressure equilibration to the waste medium container (reference pressure). At the lower end, a tube was connected to the waste medium container, allowing the medium to drain.

\section{Fluid system}

As described above, the measurements in the recording chambers required top pressure to the oocytes to be set up first, followed by media exchange (Figure 2), which was driven by air pressure. The supply air pressure, which was stored in two gas cartridges, was reduced and regulated to the appropriate pressure levels by three pressure controllers: perfusion pressure, top pressure, and waste pressure. The perfusion pressure was responsible for the medium exchange in contact with the cell membrane patch. The top pressure kept the oocytes in place and ensured an adequate seal at the membrane patch. Since the experiment was exposed to vacuum conditions during the flight, it had to be implemented as a closed system. As liquid was transferred from the fresh medium container through the recording chambers into the waste medium container, the pressure in the fluid system increased gradually. Therefore, a third pressure controller released the pressure from the waste container. The waste pressure also acted as the reference pressure for the entire fluidic system and was controlled by an absolute pressure controller (referring to absolute pressure). The pressure controllers for the top and perfusion pressures were relative pressure controllers, which adjusted the pressure so that it was always relative to the pressure in the waste container. This design made it possible to purchase all pressure controllers in the smallest possible working range, thereby providing the highest accuracy. It also ensured that the pressure differences in the recording chambers (between top and waste pressure and between perfusion and waste pressure) were always regulated accurately. The nominal top pressure was $30 \mathrm{mbar}$, and the nominal perfusion pressure was $150 \mathrm{mbar}$. The waste pressure was kept at 1 bar(a).

The fresh and waste medium containers were equipped with custom-made plastic bags. Medium could be pushed in and out of the bags by controlling the pressure of the gas phase outside of the bags. Fresh medium was pushed by the perfusion pressure out of its container and subsequently distributed via silicone tubes to the two late access modules (Figure 2). In each late access module, the medium lines were split again, leading to the valves that controlled the flow of medium through the chamber. In order to ensure a constant flow, the medium then passed through a $40 \mathrm{~cm}$-long capillary with an inner diameter of $0.18 \mathrm{~mm}$. After the medium passed through the recording chamber, it continued to run through a short tube to the waste medium valve, which was closed during launch and in case of malfunction. Directly after the recording chamber, a $5 \mathrm{~mm}$-long capillary was placed in the tube, generating a small amount of back-pressure. From the waste medium valve, the waste medium was brought to an intermediate waste medium container that was mounted on the front side of the late access module. From there, the medium was finally collected in the waste medium container. 


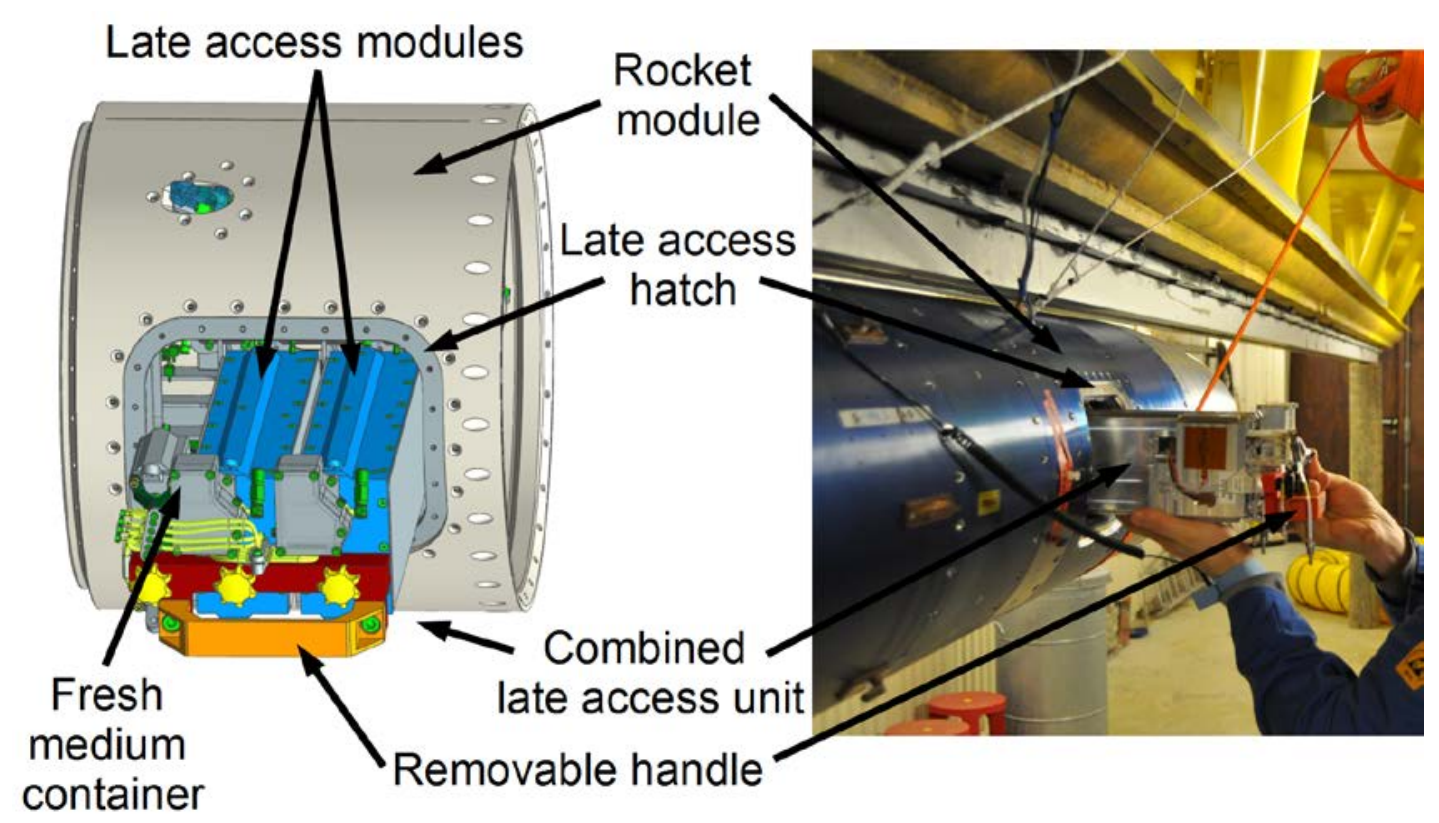

Figure 6. Late access module insertion. To keep the oocytes as fresh as possible, they were inserted into the rocket shortly before launch. Two late access modules and the fresh medium container were inserted through a late access hatch. These three elements were prepared in the laboratory before launch and combined into a single unit with a removable handle; the handle was removed before the hatch was closed. The connection of the liquid tubes was made with quick dry connectors. The electrical connections were standard D-sub connectors, which automatically made electrical contact when the late access module was inserted. The left panel is a schematic of a computer model to show how the late access module was inserted into the rocket module. The right panel is an image of the late access shortly before launch.

The pressure in the recording chamber outlet had to be kept constant and equal to the pressure in the waste medium container (reference pressure). If this pressure increased, the oocyte would be displaced (i.e., the seal of the membrane patch would break). In contrast, if the pressure decreased (e.g., through suction), the oocyte would be aspirated through the aperture in the silicone chip and lost. Due to the hydrostatic pressure difference, large elevation drops from the recording chamber to the waste medium container had to be avoided. However, the limited availability of space forced the installation of the waste medium container at the bottom of the experiment hardware. This issue was solved by introducing an intermediate waste medium container that was mounted on the front side of the late access module, illustrated in Figure 5. The waste lines from the recording chambers entered the upper part of this container, ensuring that under normal conditions, the exit of these tubes was always in contact with the gas phase, but never with the liquid phase. A further connection located in the upper part ensured that the pressure in the container was constant and independent of the filling level. This connection enabled fast pressure equilibration to the waste medium container, which was the reference pressure for the entire fluidic system. Under normal conditions, pressure was equilibrated only through the gas phase. Finally, at the lower end of this intermediate waste medium container, a tube was connected to the waste medium container, allowing the medium to drain.

\section{Late access}

In order to keep the living oocytes as fresh as possible, the oocytes were inserted into the rocket shortly before launch. This required a socalled late access hatch in the rocket through which the two late access modules and the fresh 
medium container were inserted. These three elements were prepared in the laboratory before launch and then combined into a single unit with a removable handle, illustrated in Figure 6; the handle was removed after insertion into the rocket. The connection of the liquid tubes was made with quick-dry connectors, and the electrical connections were made using standard D-sub connectors, which made electrical contact automatically when the late access module was inserted.

\section{Electronics and Software}

Figure 7 demonstrates how the electronics were distributed across four PCBs: one main board for the computer, two data acquisition units, and one power supply unit. For the interconnection of these PCBs, a Controller Area Network (CAN bus) was used due to its insensitivity to electromagnetic interference and high reliability.

The board computer was the controlling unit for the experiment, and it was responsible for correctly executing the experimental protocol, controlling the pressure regulators, saving the measured data on two redundant nonvolatile storage devices (SD-card and flash drive), and communicating with the rocket's service module. The measurement units were controlled through the CAN bus by the board computer. In addition to the electrophysiological readings, the acceleration was measured and recorded by two 3-axis accelerometers with a range of $24 \mathrm{~g}$ and $2 \mathrm{~g}$, respectively.

The measurement units controlled the applied voltages, measured the corresponding current and effective applied voltage, switched the valves controlling the media flow and the top pressure, and recorded the temperature in the recording chambers. While still on the ground, the units could also switch on heat foils under the recording chambers in case the temperature of the recording chambers dropped. The measurement units were directly mounted in the two late access modules in close proximity to the recording chambers. Commands from the board computer were received via the CAN bus and processed by a microcontroller. Measurement data was transmitted continuously over the CAN bus back to the board computer where they were saved. The microcontroller on the measurement units mastered three digital-toanalog converters. while the measured voltages and currents were digitized by six analog-todigital converters. The temperature in the recording chambers was acquired with a digital temperature sensor in each of the three chambers. These temperature readings were sent to the board computer and were also used to control heating foils under the recording chambers.

The rocket's service module provided the experiments with power, inflight signals, and an RS422 serial communication interface. The $28 \mathrm{~V}$ power supply from the service module was converted by the power supply unit to the various voltage levels required. In addition, three signals were submitted during countdown and the flight. They were used to synchronize the procedures executed by the board computer with the major flight events. The first signal, "Start of Data Storage," was submitted two minutes before liftoff and prepared the experiment for launch. The second signal, "Liftoff," signaled motor ignition, as the name implies. After receiving the "Liftoff" signal, the board computer reset the timer. The last signal, "Start of Experiment," was submitted after motor separation and indicated the start of the microgravity phase. The serial communication interface enabled the data downlink during the flight. Due to the limited bandwidth, the acquired data was down-sampled by the board computer. While the rocket was still on the ground, the experiment could also be commanded via uplink. This allowed for final system level checks during the countdown. The successful insertion of the late access module could also be confirmed via uplink commands.

\section{Heating System and Thermal Design}

In order to keep the living oocytes healthy, the temperature had to stay between $10^{\circ} \mathrm{C}$ and $20^{\circ} \mathrm{C}$ (Goldin, 1992). In addition, the air temperature inside the module had to stay above $0^{\circ} \mathrm{C}$ to prevent the medium from freezing inside the thin transporting tubes. The walls of the module were covered with an insulation layer and a polycarbonate plate separated the aluminum structure from the bulkhead. Heating foils were also installed under each recording 
chamber. The polyimide heating foils were glued onto the aluminum late access module, and an additional foil was placed on the fresh medium container. Each heating foil provided $10 \mathrm{~W}$ of heating power. Three control loops were implemented, one in each late access module and one for the fresh medium container. A two-position controller was implemented in the software of the measuring units and the board computer. The power for heating was provided on the ground by an umbilical cord that automatically disconnected during launch. Thereafter, heating was no longer possible. Because the rocket's surface heated up during ascent, and due to the short flight duration, no additional heating was necessary.

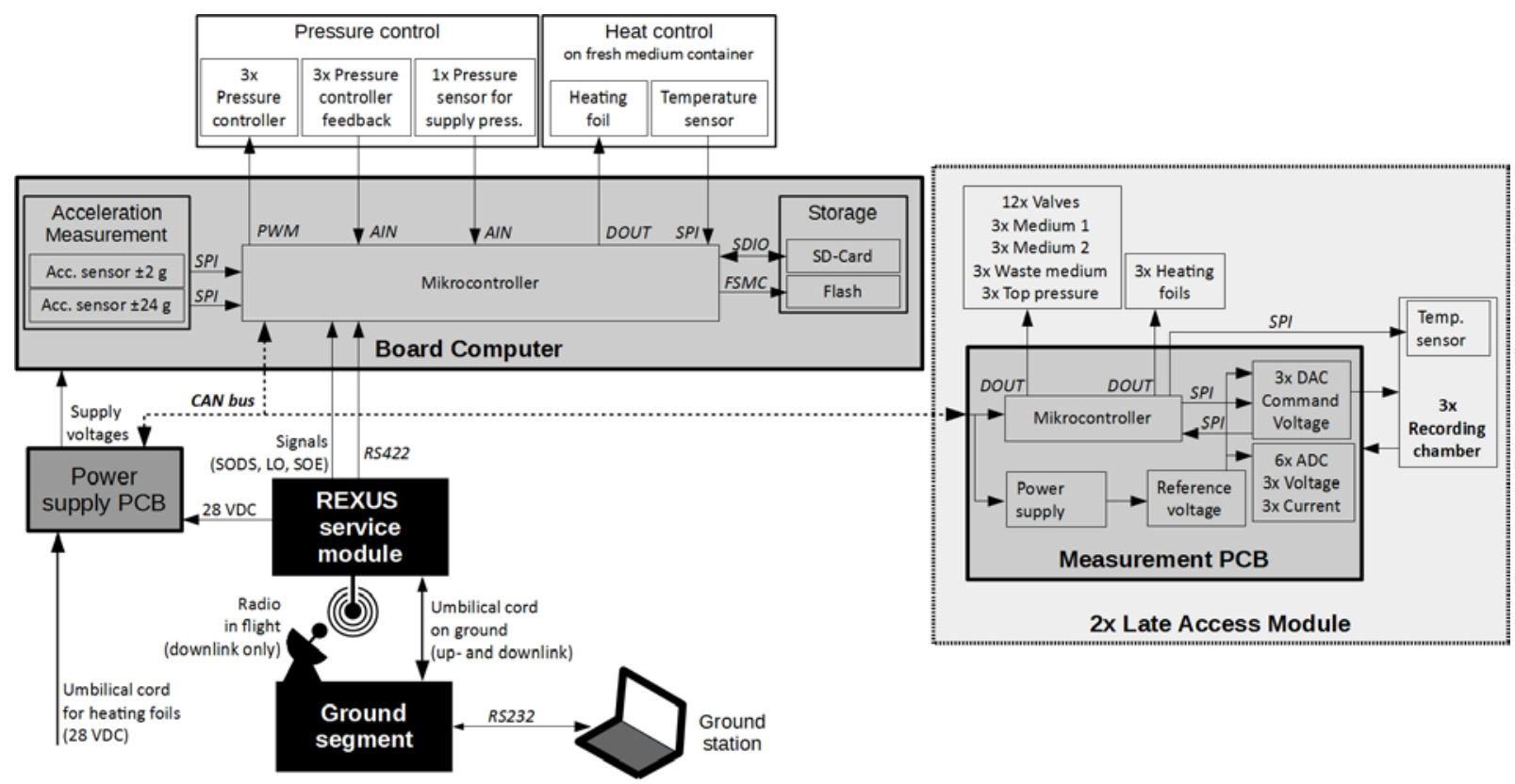

Figure 7. Schematic illustration of the electronics. The electronics were distributed across four PCBs that were interconnected by a CAN bus: one main board for the board computer, two measurement units, and one power supply unit. The board computer was the controlling unit of the experiment and was responsible for correctly executing the experiment protocol, saving the measured data (on two nonvolatile storage devices), controlling the pressure regulators, recording the acceleration, and communicating with the rocket's service module. The measurement units controlled the voltages applied to the oocytes and measured the effective applied voltage and the corresponding current. Further, they switched the valves and recorded the temperature (temp.) in the recording chambers. While still on the ground, they also controlled heating foils under the recording chambers should the chambers become too cold. The rocket's service module provided the experiments with power, inflight signals, and an RS422 serial communication interface. The $28 \mathrm{~V}$ power supply from the service module was converted by the power supply unit to the various voltage levels required. Three signals were submitted during the countdown and flight to synchronize the board computer with the major flight events. The serial communication interface allowed for data downlink during the flight and commanding via uplink while the rocket was still on the ground.

\section{Chemicals and Oocytes}

Native Xenopus laevis oocytes were obtained from the University of Zürich (Switzerland) and Ecocyte (Castrop-Rauxel, Germany). The oocytes were prepared by the respective organizations and transported fresh to the launch site at ESRANGE (Kiruna, Sweden). The oocytes were kept at $17^{\circ} \mathrm{C}$ in modified Barth medium (Goldin, 1992), containing $88 \mathrm{mM} \mathrm{NaCl}, 1 \mathrm{mM} \mathrm{KCl}, 0.41 \mathrm{mM} \mathrm{CaCl}$, $0.82 \mathrm{mM} \quad \mathrm{MgSO}_{4}, 2.5 \mathrm{mM} \mathrm{NaHCO}_{3}, 2 \mathrm{mM}$ $\mathrm{Ca}\left(\mathrm{NO}_{3}\right)_{2}$, and $7.5 \mathrm{mM}$ HEPES-TRIS, adjusted to a $\mathrm{pH}$ of 7.5. Oocytes were selected visually 
under the microscope just before preparing the late access module.

To demonstrate the rapid exchange of medium that was in contact with the cell membrane patch, either $100 \mathrm{Na}$ or $100 \mathrm{Ch}$ medium was used. The $100 \mathrm{Na}$ medium contained $100 \mathrm{mM} \mathrm{NaCl}, 2 \mathrm{mM} \mathrm{CaCl} 2,1 \mathrm{mM}$ $\mathrm{MgCl}_{2}, 2 \mathrm{mM} \mathrm{KCl}$, and $10 \mathrm{mM}$ HEPES-TRIS ( $\mathrm{pH}$ buffer). In contrast, the $100 \mathrm{Ch}$ medium contained $100 \mathrm{mM}$ choline chloride $(\mathrm{ChCl}), 1$ $\mathrm{mM}$ Ethylenediaminetetraacetic acid (EDTA), and $10 \mathrm{mM}$ HEPES-TRIS. When switching from the more usual $100 \mathrm{Na}$ medium (which was also the bath medium in the upper compartment of the recording chamber) to the $100 \mathrm{Ch}$ medium, native oocytes showed a small and unspecific reaction, because $100 \mathrm{Ch}$ contains a very small amount of cations. This reaction is illustrated in Figure 8.

\section{Sounding Rocket and Flight}

The REXUS 20 rocket was equipped with an "improved Orion" motor and had four experiments on board. REXUS 20 weighed $545.8 \mathrm{~kg}$ and measured $5.96 \mathrm{~m}$ in length, which is particularly long and heavy compared to usual REXUS rockets. Therefore, at $77.5 \mathrm{~km}$, the apogee was lower than under normal conditions. The acceleration and altitude profiles are depicted in Figure 9. The late access module was prepared shortly before launch and was inserted into the rocket just before arming. The successful insertion was checked via the uplink/downlink communication. Oocyte integrity was checked again minutes before liftoff.

\section{RESULTS}

The CEMIOS experiment was developed and conducted within the REXUS/BEXUS program (Callens et al., 2013), which is maintained under a bilateral agreement between the German Aerospace Center (DLR) and the Swedish National Space Board. It was conducted in March 2016 on the REXUS 20 rocket under almost optimal conditions. As the flight was scheduled for early morning, the late access modules were prepared during a night shift. All the systems of the experiment operated as planned during the flight. The temperature in the recording chamber was stable between $15^{\circ} \mathrm{C}$ and $18^{\circ} \mathrm{C}$. The temperature recorded on the fresh medium container increased during the flight, but remained in an acceptable range between $14^{\circ} \mathrm{C}$ and $17^{\circ} \mathrm{C}$. Additionally, no significant pressure drop was recorded, which would have indicated a major leak. Furthermore, we assessed if the medium in contact with the membrane patch was exchanged by switching between the different media $(100 \mathrm{Na}$ and $100 \mathrm{Ch}$ ) as explained previously. This test worked well during the test run shortly before launch (Figure 8).

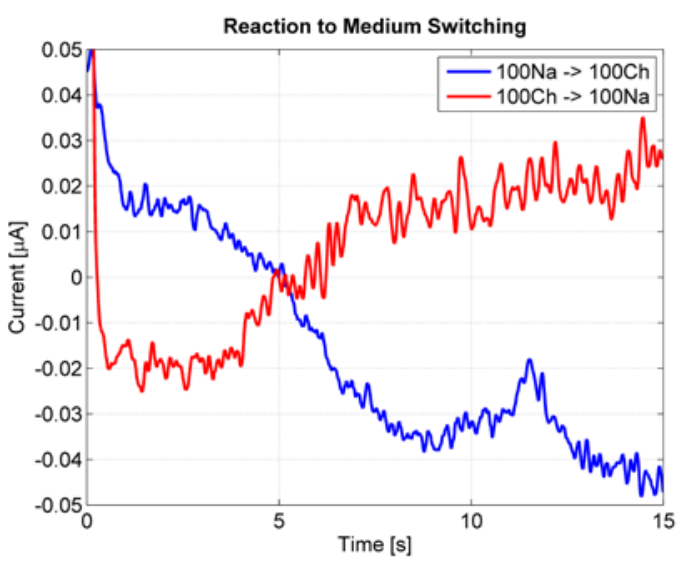

Figure 8. Graph depicting the current signal after medium switches. The oocyte membrane patch was normally perfused with $100 \mathrm{Na}$. When medium was switched from $100 \mathrm{Na}$ to $100 \mathrm{Ch}$ and back, a small and unspecific reaction was provoked from native oocytes, likely since $100 \mathrm{Ch}$ possesses very few cations. The graph indicates the current signal after switching from $100 \mathrm{Na}$ to $100 \mathrm{Ch}$ (blue line), and from $100 \mathrm{Ch}$ to $100 \mathrm{Na}$ (red line). The signals were shifted such that 0 seconds indicates the switching events. The holding voltage across the oocyte was kept at $0 \mathrm{mV}$.

However, as the rocket reached the microgravity phase, all of the recording chambers appeared to be empty. Data from the launch period, exhibited in Figure 10, showed a sharp drop in the applied voltage, accompanied by a sharp rise in the current signal just a few seconds into the flight. This indicates a sudden short circuit between the upper and lower compartments (or electrodes) in the recording chamber. These events likely indicate the time points when the oocytes were disrupted. 
Inspection of the hardware after the flight revealed that the waste compartments were full of oocyte debris, which is consistent with the destruction of the oocytes. Because of this issue, no electrophysiological recordings were possible under the microgravity conditions.

\section{DISCUSSION}

Since all recording chambers were empty by the microgravity phase, we were not able to demonstrate the feasibility of the proposed electrophysiological experiments. The voltage and current readings during the launch suggest that the oocytes were ripped a few seconds into the flight, which we believe were caused by the vibrations that occurred at launch. Ground tests, performed during the experiment validation phase, demonstrated that survival of the oocyte is critical during vibration. The recording chambers were fitted with foam to dampen vibrations, which was sufficient for ground based vibration tests (random vibration along a single axis at $12.7 \mathrm{~g}_{\mathrm{RMS}}$ ). It is therefore possible that the linear acceleration during the launch compressed the foam, thereby reducing its damping properties.

\section{REXUS 20}
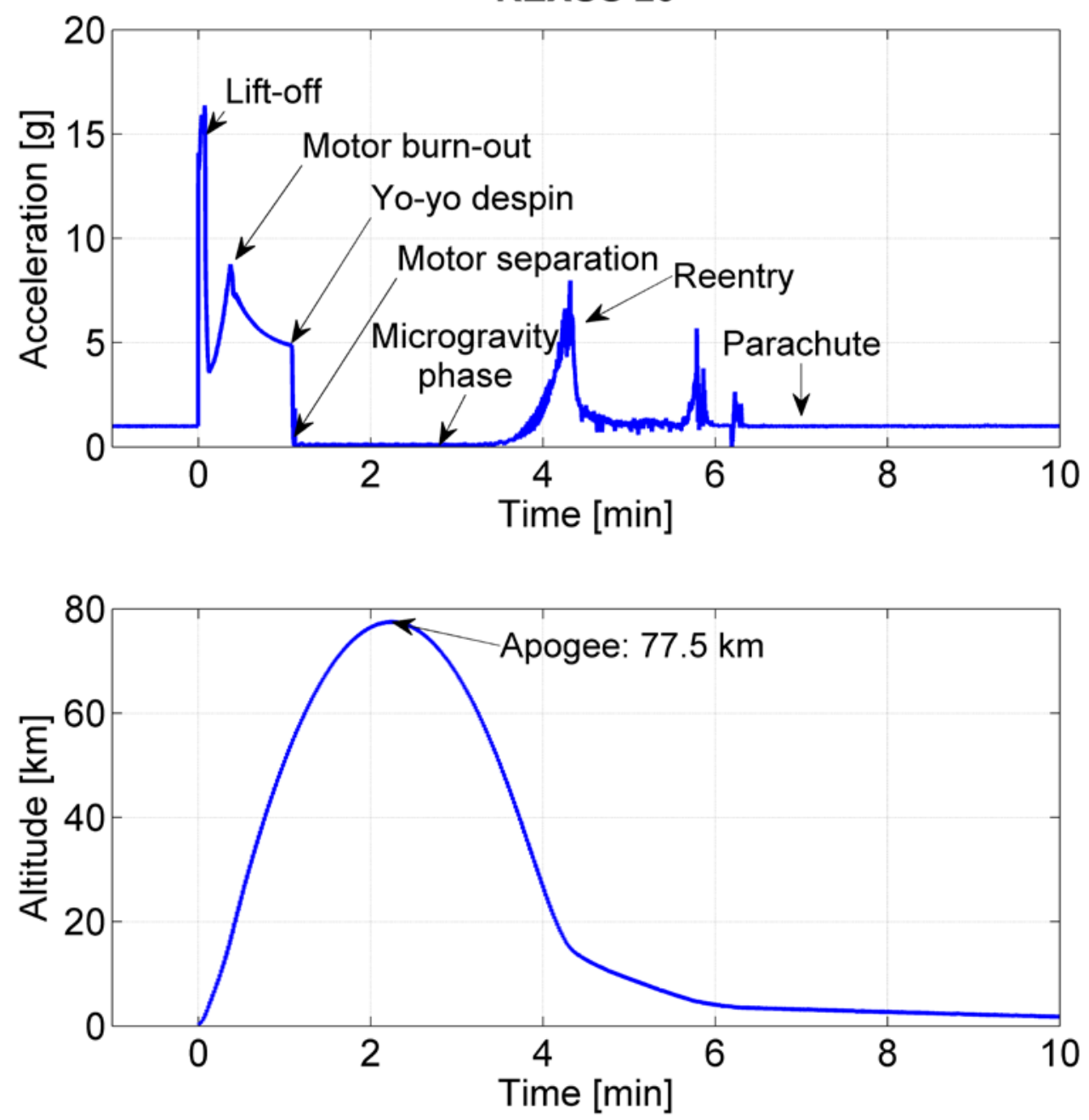

Figure 9. The acceleration and altitude profiles recorded by the REXUS service module. The top graph shows the acceleration profile with the major flight events indicated by arrows. The bottom graph shows the altitude profile. Because REXUS 20 was longer and heavier than previous REXUS rockets, the apogee was lower than usual. Data were provided by the Mobile Rocket Base (MORABA) from the German Aerospace Center (DLR). 

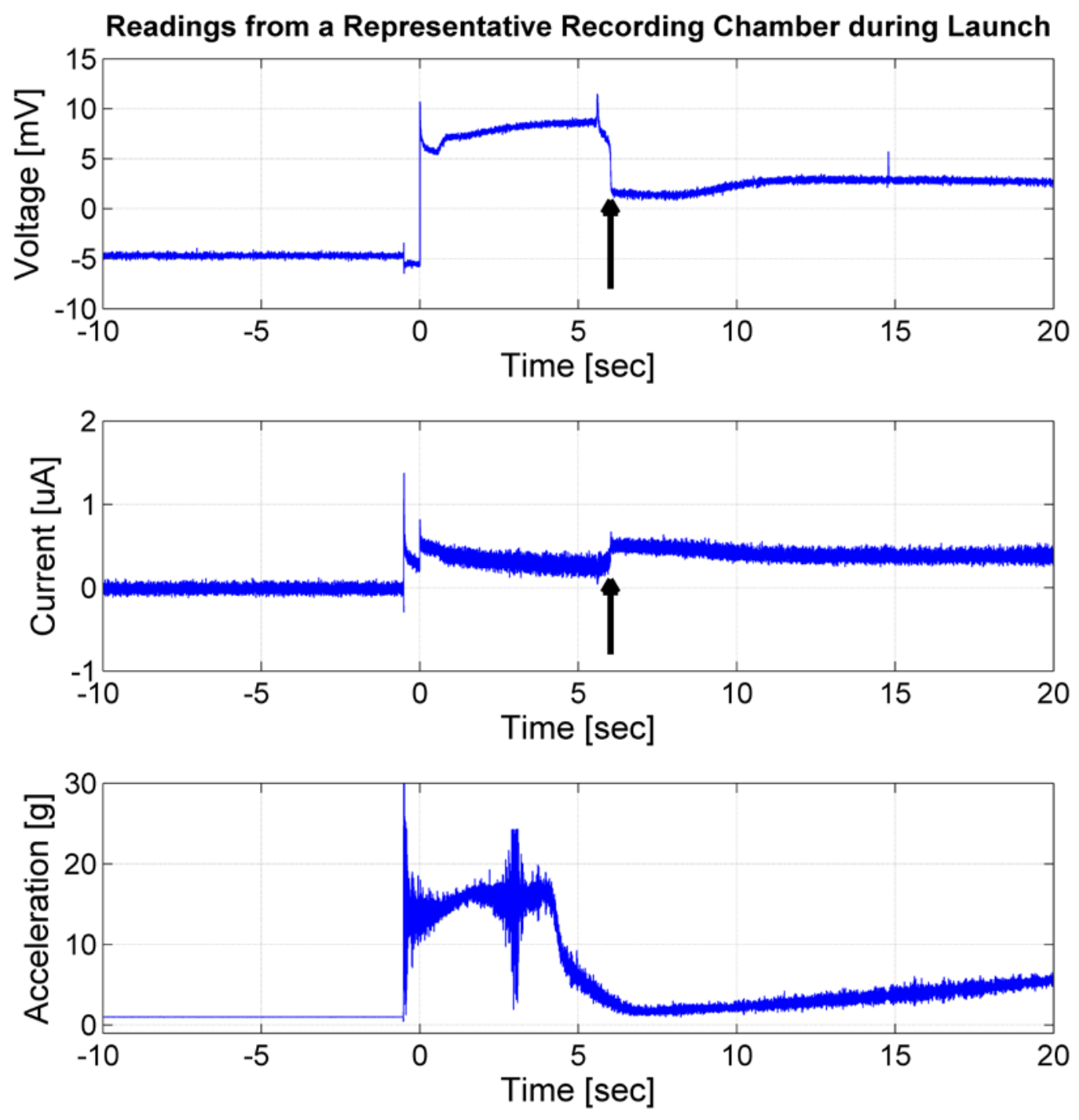

Figure 10. Representative reading of a recording chamber during launch (liftoff at 0 seconds). The board computer triggered a voltage jump at liftoff to assess whether the liftoff signal was received and to obtain a measurement of the seal resistance. A sharp drop in the applied voltage, accompanied by a sharp rise in the current signal, was observed a few seconds into the flight (arrow). Since the recording chambers all appeared empty as the rocket reached the microgravity phase, this event likely indicates the time point when the oocytes were ripped apart.

In addition, there were several critical issues attached to this electrophysiological experiment that affected the viability of the oocytes before the launch even occurred. First, although the recording chamber was designed to provide optimal conditions for the oocytes, they did not always survive; some died within an hour, while others survived several hours. Unfortunately, we were not able to predict whether the oocytes would do well in the recording chambers by visual inspection for morphological specifications alone. Although the flight duration was approximately $10 \mathrm{~min}$, the preparation and late access took several hours, during which the oocytes had to survive in the recording chamber. Second, due to the high 
level of integration of the experiment hardware, replacing a lost oocyte could take up to 30 minutes, which disrupted the experiment, particularly when close to launching the sounding rocket. Third, for safety and operational reasons, it was often not possible to perfuse and monitor the oocytes, which reduced their survival chances. Finally, the late access module had to be inserted while the rocket was in a horizontal position, which risked oocyte displacement during late access and the subsequent arming procedure. For these reasons, three oocytes were lost after arming, despite extensive practice and simulation of the loading procedure.

In conclusion, electrophysiological measurements aboard a sounding rocket are difficult to perform under the conditions presented here, and further improvements on the experimental design and operations will need to be implemented before a reflight. Given the results from the REXUS 20 flight, several questions must be answered: What vibrations (frequency and magnitude) can oocytes tolerate? What kind of stress (acceleration and/or vibration) caused the final rupture of the oocytes at launch? In lieu of visual screening, how can we better screen oocytes to ensure the recording chamber is equipped with only the fittest oocytes? It should also be possible to apply this method at the launch facility. A general challenge, however, was that insertion of the late access module had to occur while the sounding rocket was in a horizontal position, which made the design of the module, including the recording chamber, more complicated. It would significantly help the experiment if the late access could be performed after the erection of the rocket.

\section{ACKNOWLEDGEMENTS}

We acknowledge our internal supporters, namely Ralf Baumann and Marcel Joss. We thank Ian Forster and Eva Hänsenberger from the University of Zürich for providing us with oocytes and providing their expertise. We also thank Benjamin Gantenbein from the University of Bern and the Gebert Rüf project \# GRSX028/13 for supporting this project. We thank the supporters from the industry, namely Ruag
Thun, Plastika Balumag AG, and Plüss AG. We furthermore thank the Swiss Space Office for financing this project. Finally, we thank the REXUS/BEXUS program and its supporting agencies, namely the DLR, the Swedish National Space Board, and the European Space Agency for making this project possible.

\section{CONFLICT OF INTEREST}

The authors declare that they have no conflicts of interest.

\section{REFERENCES}

Callens N, Kinnaird A, Dannenberg K, Fittock $\mathrm{M}$, Inga $\mathrm{M}$, Persson $\mathrm{O}$, Roth $\mathrm{M}$, Schmitdt A, Siegl M (2013) REXUS/BEXUS Rocket and balloon experiments for university students. 21st ESA Symposium on European Rocket \& Balloon Programmes and Related Research 9-13 June 2013. Thun, Switzerland: European Space Agency

Eyckmans J, Boudou T, Yu X, Chen CS (2011) A hitchhiker's guide to mechanobiology. Developmental Cell 21: 35-47

Goldermann M, Hanke W (2001) Ion channel are sensitive to gravity changes. Microgravity Science and Technology 13: 35-38

Goldin AL (1992) [15] Maintenance of Xenopus laevis and oocyte injection. In Methods in Enzymology Vol. 207, pp 266-279. Academic Press

Hanke W, Fernandes de Lima MV, Wiedemann M, Meissner K (2006) Microgravity dependence of excitable biological and physicochemical media. Protoplasma 229: 235-242

Hanke W, Wiedemann M, Fernandes de Lima VM (2002) Control of the excitability of neuronal tissue by weak external forces. Faraday Discussions 120: 237-248

Kohn FPM (2013) High throughput fluorescent screening of membrane potential and intracellular calcium concentration under variable gravity conditions. Microgravity Science and Technology 25: 113-120

Meissner K, Hanke W (2005) Action potential properties are gravity dependent. 


\section{Wuest et al. -- Electrophysiological Recordings on a Sounding Rocket}

Microgravity Science and Technology 17: 38-43

Pietsch J, Bauer J, Egli M, Infanger M, Wise P, Ulbrich C, Grimm D (2011) The effects of weightlessness on the human organism and mammalian cells. Current Molecular Medicine 11: 350-364

Richard S, Henggeler D, Ille F, Vadrucci Beck S, Moeckli M, Forster IC, Franco-Obregón A, Egli M (2012) A semi-automated electrophysiology system for recording from Xenopus oocytes under microgravity conditions. Microgravity Science and Technology 24: 237-244

Schaffhauser DF, Andrini O, Ghezzi C, Forster IC, Franco-Obregon A, Egli M, Dittrich PS (2011) Microfluidic platform for electrophysiological studies on Xenopus laevis oocytes under varying gravity levels. Lab on a Chip 11: 3471-3478

Sieber M, Hanke W, Kohn FPM (2014) Modification of membrane fluidity by gravity. Open Journal of Biophysics 4: 7

Wiedemann M, Fernandes de Lima VM, Hanke W (2002) Gravity dependence of waves in the retinal spreading depression and in gel type Belousov-Zhabotinsky systems. Physical Chemistry Chemical Physics 4: 1370-1373

Wiedemann M, Hanke W (2002) Gravity sensing in the central nervous system. Journal of Gravitational Physiology 9: P43-P44

Wuest SL, Roesch C, Ille F, Egli M (2017) Calcium dependent current recordings in Xenopus laevis oocytes in microgravity. Acta Astronautica 141: 228-236 